\title{
Hommage à Michèle Therrien
}

Pierre Rousseau

\section{(2) OpenEdition}

Journals

Édition électronique

URL : https://journals.openedition.org/jsa/17013

DOI : 10.4000/jsa. 17013

ISSN : $1957-7842$

\section{Éditeur}

Société des américanistes

\section{Édition imprimée}

Date de publication : 15 juin 2019

Pagination : 181-185

ISBN : 978-2-902715-11-4

ISSN : 0037-9174

\section{Référence électronique}

Pierre Rousseau, « Hommage à Michèle Therrien », Journal de la Société des américanistes [En ligne], 105-1 | 2019, mis en ligne le 15 juin 2019, consulté le 04 septembre 2022. URL : http:// journals.openedition.org/jsa/17013; DOI : https://doi.org/10.4000/jsa.17013 


\title{
Hommage à Michèle Therrien
}

\author{
$\mathrm{M}^{\mathrm{e}}$ Pierre Rousseau *
}

J'ai connu le docteur Michèle Therrien vers 1986, alors que je travaillais au ministère de la Justice du Québec comme coordonnateur des services judiciaires en milieu autochtone. J'étais substitut du Procureur général du Québec détaché auprès des services judiciaires (soutien aux tribunaux) pour ce mandat spécifique et mon collègue $\mathrm{M}^{\mathrm{e}}$ Jacques Auger, le coordonnateur ministériel pour les questions autochtones, m'avait invité à une rencontre avec le Dr Therrien. J'étais surpris par cette invitation car il m'expliqua que le Dr Therrien enseignait l'inuktitut à Paris, à l'Institut national des langues et civilisations orientales, et j'étais étonné qu'on enseignât cette langue autochtone en France, qui plus est comme langue orientale!

Ce fut une rencontre qui changea ma vie... Comme nous devînmes de grands amis, je me permets d'appeler le Dr Therrien par son prénom. Donc, Michèle nous expliqua qu'elle s'intéressait à la justice chez les Inuit du Canada et qu'elle avait le projet d'analyser leur perception du système judiciaire canadien à partir de leurs mots pour en traduire les concepts et le vocabulaire. J'étais fasciné par cette femme exubérante qui venait du Québec mais qui avait atteint une expertise très rare sur les Inuit et qui occupait un poste très prestigieux dans une université renommée de France. À ce moment-là, personne à ma connaissance n'enseignait l'inuktitut au Canada! Nous convînmes que ce serait moi qui agirais comme personne-ressource auprès de Michèle en ce qui a trait au système judiciaire canadien et c'est ainsi qu'une longue amitié et une intense collaboration naquirent.

Les travaux de Michèle étaient très intéressants, d'autant plus que m'étant rendu à Puvirnituq - Nunavik (nom inuit du nord québécois) - pour des ateliers de formation avec le comité de justice local, le Sapuulutait, je fus très surpris quand les Inuit rirent à gorge déployée lorsque je leur expliquais que la Cour d'appel du Québec comprenait trois juges... L'interprète m'avoua qu'il avait traduit l'expression « trois juges » en inuktitut par « trois grands cous »...

* Avocat à la retraite [hprousseau@videotron.ca]. 
J'étais très étonné par cette traduction et il m'apprit que le juge de la cour itinérante qui visitait occasionnellement le village avait été surnommé en inuktitut « grand cou » à cause de ses caractéristiques physiques et qu'il ne connaissait pas d'autre mot pour « juge ». Par la suite, Michèle m'expliqua qu'il existait un mot en inuktitut pour « juge » : iqqaqtuiji. La traduction littérale de ce mot signifie « une personne qui oblige quelqu'un à se souvenir d'événements passés » ou, en d'autres mots, une personne qui oblige quelqu'un à se souvenir de mauvaises actions plutôt que des bonnes, l'aidant à exprimer une chose non dite mais dont on se souvient. Ce fut une véritable révélation car je compris alors que les Inuit reprochaient au système judiciaire de les obliger à se souvenir de mauvaises actions, ce qui entrait en conflit avec leur culture qui vise plutôt à réparer les torts causés et rétablir l'harmonie dans la collectivité tout en effaçant la mauvaise action.

En 1994, avec mon appui alors que j'étais directeur régional du bureau des Territoires du Nord-Ouest à Yellowknife pour le ministère de la Justice du Canada, Michèle fit une demande de financement auprès du gouvernement canadien pour poursuivre ses recherches. Elle venait de conclure un projet semblable au Nunavik sur la santé et la maladie avec l'aide d'un aîné très respecté de Puvirnituq, Taamusi Qumaq. Simultanément, elle avait commencé ses travaux sur la justice et elle avait déjà un lexique de deux cents mots. Avec Susan Inuaraq, une Inuk qui travaillait à mon bureau d'Iqaluit comme parajuriste ${ }^{1}$, elles voulaient démontrer que l'inuktitut, qui utilise plusieurs mots pour décrire une seule réalité, avait beaucoup à dire à propos de la justice, contrairement à ce que croyaient les gens de la société dominante. Il s'agissait de:

- identifier les raisons pour lesquelles les Inuit ont tellement de difficultés

à comprendre le système juridique canadien;

- reconnaitre les traditions juridiques des Inuit;

- encourager la participation des aînés en leur donnant un rôle officiel en tant que consultants. C'est essentiel pour un vrai dialogue entre les Inuit et les Qallunaat (les membres de la société dominante de langue anglaise); - encourager tous les décideurs, y compris ceux du ministère de la Justice, à tenir compte des valeurs des Inuit, entre autres, en organisant des ateliers interculturels pour discuter des résultats du projet;

1. Ce terme désigne les personnes qui travaillent comme professionnels dans le domaine juridique mais qui n'ont pas de diplôme universitaire de droit et ne sont pas avocats. En anglais, elles sont désignées comme étant des paralegal. Grâce, notamment, aux encouragements et au soutien de Michèle, Susan réussi à obtenir son baccalauréat en droit en 2005 à la faculté de droit Akitsiraq, dans le cadre d'un programme conjoint entre l'université de Victoria (Colombie-Britannique) et le Collège de l'Arctique du Nunavut qui comprenait un volet sur le droit traditionnel inuit. 
- aider les Inuit à avoir une meilleure connaissance du processus des tribunaux et aider les juges, les avocats et les autres professionnels à comprendre les collectivités;

- aider les traducteurs, les interprètes, les étudiants et tous les autres Inuit qui travaillent quotidiennement au sein du système judiciaire.

Malheureusement, le gouvernement de l'époque portait peu d'intérêt aux questions autochtones malgré le fait que la Commission royale sur les peuples autochtones poursuivait alors ses travaux à travers le pays et allait soumettre son rapport final deux ans plus tard, rapport dont les quelque quatre cent quarante recommandations furent ignorées par ce gouvernement et les autres qui suivirent jusqu'à maintenant.

Cela ne découragea pas Michèle, loin de là. Elle organisa plusieurs conférences sur le monde inuit à Paris et contribua à plusieurs autres un peu partout dans le monde. Elle m'invita à participer à quelques-unes d'entre elles entre 1993 et 2004. J'y abordai les questions de lois et de justice à l'aube de la naissance du Nunavut et des conflits culturels entre la société inuit et le système judiciaire canadien. À l'occasion je fus accompagné d'une Inuk, invitée aussi par Michèle, qui expliqua certains éléments de la « justice» traditionnelle inuit, comme mode de contrôle social. Cette présentation nous a permis de comprendre la nature des systèmes juridiques inuit et leur fonctionnement, ce qui fut une découverte pour plusieurs d'entre nous.

On y traita aussi des questions entourant la mise en place du Nunavut et des espoirs déçus car il existait alors une atmosphère de crise dans le nouveau territoire. La question des conflits interethniques entre la société dominante, plutôt coloniale, et les Inuit fit l'objet de bien des discussions dans l'espoir de trouver des moyens de les gérer et de les atténuer. Toutefois, il était devenu évident que du côté de la justice, le Canada continuait à traiter les Inuit du Nunavut comme le ferait toute autre autorité coloniale, refusant de reconnaître leurs systèmes juridiques propres et leurs lois. Dans ce contexte, la transmission intergénérationnelle du savoir traditionnel, de manière orale, dans une société nouvelle de plus en plus axée sur l'écrit posait un défi considérable pour l'inuktitut, d'où l'intérêt des travaux de Michèle en matière linguistique.

Lorsqu'elle vint à Iqaluit à l'automne 1994 pour une conférence internationale organisée par la revue Études inuit/Inuit studies, elle m'expliqua où elle en était arrivée dans ses recherches sur la justice chez les Inuit. Il ressortit de nos discussions que les Inuit percevaient le système judiciaire canadien comme un système imposé de force, d'où le mode passif de l'inuktitut pour en traduire les éléments. Par exemple, l'emprisonnement se traduit par tigujaumaniq, littéralement « être saisi par la main par quelqu'un ». Ce mode passif souligne le fait que les Inuit sont l'objet d'arrestations, d'interrogations, de jugements, de condamnations et d'emprisonnement par des tiers, dans ce cas, les Qallunaat. 
Cela illustre aussi la nouveauté et la singularité de ces procédures qui sont tellement étrangères à la culture et à l'histoire des Inuit.

Nous étions aussi très préoccupés par le concept de culpabilité. Nous savions que, dans la culture inuit, cette notion n'existait pas avant le contact avec les Euro-Canadiens. Or, quand le tribunal demandait à un accusé s'il plaidait coupable ou non coupable, nous nous sommes demandés quels étaient les mots en inuktitut qui reflétaient cette nouvelle réalité. Les traducteurs eurent beaucoup de difficultés avec ce concept et nous constatâmes que le résultat était plus ou moins satisfaisant. Le mot « coupable » se traduit par pasijaksaq qui, si on le décortique en français, se traduit littéralement par « quelqu'un qu'on soupçonne, qu'on blâme » avec la notion qu'il s'agit d'une tierce personne qui pose ce jugement, pas l'accusé. Or, ce devrait être l'accusé lui-même qui indique au tribunal s'il se considère coupable au lieu de tiers qui pourraient se tromper. À l'inverse, la traduction de « non coupable », pasijaksaunngittuq qui se traduit littéralement par « quelqu'un qu'on ne soupçonne pas, qu'on ne blâme pas » n'est pas plus satisfaisante.

Comme notre projet ne reçut aucun soutien du gouvernement canadien, Michèle dut abandonner l'idée d'explorer les deux cents mots qu'elle avait identifiés mais elle put quand même s'assurer qu'une partie de ce travail ne soit pas perdu car il se retrouve dans le dictionnaire juridique de Desmond Brice-Bennett auquel elle contribua (1997, p. 250 à 275).

Michèle s'impliqua dans le cinéma et collabora, entre autres, au film Kabloonak de Claude Massot, en tant que traductrice des dialogues de l'inuktitut au français. Dans une lettre du 11 décembre 1994, elle me raconte cette anecdote: « lorsque la belle Sipuura [Ungalaq] (Nila dans le film) invite pour la première fois Flaherty à la suivre, elle lui dit: "uvattinut ailirit, uvattinut qailirit", ce qui est plein de sens en la circonstance et qui se traduit littéralement par "chez nous vas, chez nous viens", l'opposition ailirit et qailirit est fort belle "en la circonstance". J'ai voulu traduire au plus près par "va à la maison, viens à la maison" mais le réalisateur m'a imposé: "viens dans le petit iglou"! J'ai tout fait pour que l'on sache qu'il n'y a que les chiots qui dorment dans les petits iglous. Je lui ai même dit que "viens dans le petit iglou" est équivalent à "viens dans l'utérus" (igliq lit, plateforme, igliaq "petit lit" = utérus). Rien à faire. » Elle fut par ailleurs très affectée lorsque Claude Massot se suicida. Elle m'écrivit en avril 1995: « Ce fut un grand choc dont j'ai du mal à me ressaisir... Le film n'a pas reçu l'accueil qu'il attendait, ce qui est injuste et injustifié, d'autant que je suis convaincue que le film sera re-découvert car il est tout simplement beau, juste, vrai, sans débordement lyrique. » Peu de temps après, elle me fit rencontrer la star féminine du film, Sipuura Ungalaq, qui accepta de donner des cours d'inuktitut au personnel de mon bureau de Yellowknife durant l'hiver 1995. Une autre rencontre inoubliable et extrêmement enrichissante, grâce à Michèle. 
J'ai indiqué au début que ma rencontre avec Michèle avait changé ma vie. En fait, je lui dois d'avoir commencé à comprendre un peu les Inuit et cela m'encouragea à accepter un poste à Iqaluit en 1989, comme procureur en milieu inuit. Ce fut par la suite l'expérience la plus extraordinaire de ma vie. Les travaux de Michèle - étalés sur plusieurs années, en collaboration avec plusieurs Inuit que je connaissais - m'ont aussi ouvert les yeux sur le colonialisme du système judiciaire canadien (Rousseau 2019). Je me rendis compte de l'énorme fossé entre ce système et le peuple inuit (et les autres peuples autochtones), ce qui m'encouragea à pousser ma réflexion plus loin et, finalement, à faire une maîtrise sur l'analyse et la gestion des conflits interethniques. Je comprends maintenant pourquoi le Canada vit ce drame entre les peuples autochtones et la société dominante dont le système juridique est un élément majeur. Je serai éternellement reconnaissant à Michèle de m'avoir donné la chance de gratter la surface de la culture inuit et d'en avoir tiré une profonde empathie envers ce peuple. Merci Michèle!

\section{Référence citée}

BRice-BennetT Desmond

1997 Legal glossary (English/Inuktitut/French) - Glossaire juridique (anglais/ inuktitut/français), Nunavut Arctic College, Nunatta Campus, Iqaluit.

Rousseau Pierre

2019 Une justice coloniale. Le système juridique canadien et les Autochtones. Témoignage d'un procureur de la Couronne dans l'Arctique canadien, Presses de l’Université Laval, Laval. 
